Artículo científico

Volumen 30(2):459-467. Mayo-agosto, 2019

e-ISSN 2215-3608, doi:10.15517/am.v30i2.33987

https://revistas.ucr.ac.cr/index.php/agromeso/index

\title{
Extracción de apitoxina con un colector eléctrico en Irapuato, Guanajuato, México ${ }^{1}$
}

\section{Extraction of apitoxin with an electric collector in Irapuato, Guanajuato, Mexico}

\author{
Carlos Manuel Bucio-Villalobos ${ }^{2}$, Oscar Alejandro Martínez-Jaime ${ }^{3}$
}

1 Recibido: 11 de julio, 2018. Aceptado: 16 de octubre, 2018. Este trabajo formó parte del proyecto "Evaluación del potencial de rendimiento de veneno abeja utilizando una trampa novedosa de fabricación argentina, y evaluación de la eficiencia de tres capas protectoras adicionadas a la trampa para la obtención de apitoxina limpia”, financiado por la Universidad De La Salle Bajío y se llevó a cabo en Irapuato Guanajuato, México.

2 Universidad De La Salle Bajío. Av. Universidad 602, Col. Lomas del Campestre, C.P. 37150, León, Guanajuato, México. buciovillalobos@ yahoo.com.mx

3 Universidad de Guanajuato. Km 9 carretera Irapuato-Silao, C.P. 36821, Irapuato, Guanajuato, México. oscarja@ugto.mx (https://orcid. org/0000-0001-8328-5686).

\section{Resumen}

Introducción. La toxina (apitoxina) producida por abejas (Apis mellifera), es un producto con expectativas importantes en la industria médica, debido al uso terapéutico en diversas enfermedades. Objetivo. El objetivo del presente estudio fue estimar el potencial de producción anual de apitoxina, recolectada mediante un equipo automatizado funcionando con impulsos eléctricos de $10 \mathrm{~s}$ de duración y un voltaje de $12 \mathrm{v}$. Materiales y métodos. Se utilizaron cinco colmenas de un apiario localizado en Irapuato, Guanajuato, México, donde se cosechó la apitoxina de agosto de 2016 a junio de 2017, a intervalos quincenales durante un lapso de 20 min por vez, y se suspendió la colecta en aquellos periodos con flujos abundantes de néctar, con la finalidad de no afectar la producción de miel. Resultados. De acuerdo con las condiciones en las que se desarrolló este estudio, el promedio ( \pm desviación estándar)

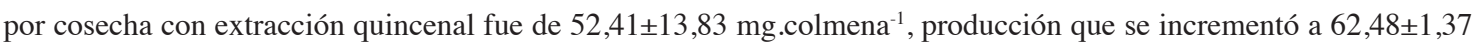
mg.colmena ${ }^{-1}$, al considerar solo colonias con bajos niveles de infestación de Varroa destructor. Conclusión. La región apícola donde se desarrolló esta investigación presentó potencial para la producción de apitoxina, proponiéndose como un producto apícola adicional que permite incrementar la rentabilidad del apiario.

Palabras clave: apicultura, productos de la colmena, toxinas.

\begin{abstract}
Introduction. The toxin (apitoxin) produced by Apis mellifera bees, is a product with important expectations in the medical industry, due to the therapeutic use in various diseases. Objetive. The objective of the present study was to estimate the annual production potential of apitoxin, collected through an automated equipment operating with electrical impulses of $10 \mathrm{~s}$ of duration and a voltage of $12 \mathrm{v}$. Materials and methods. Five beehives were used from a beekeeping farm located in Irapuato, Guanajuato, Mexico, where the apitoxin was harvested from August 2016 to June 2017, at biweekly intervals for a period of $20 \mathrm{~min}$ at a time, suspending the collection in those periods with abundant flows of nectar, to not affect the production of honey. Results. According to the conditions in which
\end{abstract}


this study was developed, the average ( \pm standard deviation) per harvest with biweekly extraction was $52,41 \pm 13,83$ mg.hive ${ }^{-1}$, production that was increased to $62,48 \pm 1,37 \mathrm{mg}$.hive ${ }^{-1}$, considering only colonies with low levels of Varroa destructor infestation. Conclusion. The apicultural region where this research was developed, presented potential for the production of apitoxin, being proposed as an additional apicultural product that allows increasing the profitability of the apiary.

Keyword: apiculture, hive products, toxins.

\section{Introducción}

La apitoxina de la especie Apis mellifera en sus diferentes razas, ha sido ampliamente utilizada con fines terapéuticos en varios países, especialmente para el tratamiento de enfermedades como la artritis, artritis reumatoide, esclerosis múltiple, lupus, dolor del nervio ciático en la espalda baja, etc.; además, se ha incluido en una práctica conocida como apiterapia (Fratellone y Fratellone, 2016), ya que a sus componentes se les han encontrado efectos analgésicos, antiinflamatorios, antiartríticos, respuestas de inmunidad, antitumorales y efectos citotóxicos contra células cancerosas, entre otros (Eze et al., 2016; Shimpi et al., 2016).

El aguijón de las abejas es una estructura localizada al final de la parte posterior del abdomen, cuando se usa para picar, este se pierde por desprendimiento, provocándose con ello la muerte de la abeja en un período de pocas horas, debido a la ruptura masiva de sus partes abdominales. Dicha estructura está compuesta de dos lancetas barbadas sostenidas en dos placas endurecidas y fuertes músculos, además de estar conectadas a varias glándulas productoras de veneno y feromonas de alarma (Winston, 1987). Aunque las reinas tienen aguijón, son las abejas obreras las que lo utilizan en su función de defender la colonia, y son dos glándulas las responsables de sintetizar el veneno, el cual es inyectado en la víctima desde el momento de la picadura y hasta de 30 a 60 segundos después del desprendimiento del aguijón (Goodman, 2003). Una abeja obrera madura es capaz de inyectar entre 0,15 y 0,30 $\mathrm{mg}$ de veneno durante el proceso de una picadura (Schumacher et al., 1989), siendo la dosis letal media estimada para humanos de 2,8 mg.kg-1 de peso (Eze et al., 2016).

Se han efectuado un gran número de estudios para dilucidar la composición química de la apitoxina, y se han detectado al menos dieciocho compuestos farmacológicamente activos (Eze et al., 2016). Se trata de un líquido trasparente con un $\mathrm{pH}$ ácido $(4,5$ a 5,5), que al hacer contacto con el aire se seca rápidamente, formando cristales de color blanco grisáceo (Mahmoud, 2012; Shimpi et al., 2016). Los componentes de la apitoxina son principalmente una mezcla de proteínas y péptidos, la melitina es el más importante, pues alcanza hasta el $52 \%$ de su peso seco, otros compuestos involucrados son: la fosfolipasa A, hialuronidasa, apamina, histamina, dopamina y adolapina (Sobral et al., 2016). Al analizar el veneno se detectaron las enzimas: fosfolipasa A2, fosfolipasa B, hialuronidasa, fosfatasa y aglucosidasa; las proteínas y péptidos: melitina, apamina, scapina, pamina, minimina, adolapina, procamina A y B, un inhibidor de la proteasa y la tertiapina; las aminas: histamina, dopamina y norepinefrina; los carbohidratos: glucosa y fructosa; los aminoácidos: ácido amino butírico y alfa aminoácidos; los minerales: fósforo, calcio y magnesio; así como algunos fosfolípidos e ingredientes volátiles (Shimpi et al., 2016). La compleja naturaleza de la apitoxina puede deberse a la amplia gama de insectos y vertebrados que actúan como plagas o depredadores de las abejas; así por ejemplo, las histaminas pueden no ser tóxicas para los vertebrados, pero sí para algunos insectos (Winston, 1987).

Las reacciones de los humanos al piquete de abeja pueden presentarse en tres niveles: local, sistémico y anafiláctico. En el primer caso, los síntomas se localizan en la zona de la picadura y corresponden a una hinchazón inmediata, que puede aumentar en pocas horas o días siguientes, es acompañada de enrojecimiento alrededor del área 
picada con una sensación de ardor, estos síntomas desaparecen en dos o tres días. Por su parte, la reacción sistémica generalmente ocurre a los pocos minutos de la picadura y se puede manifestar con sarpullido en todo el cuerpo, respiración jadeante, náuseas, vómitos, dolor abdominal y desmayo. Por último, la reacción anafiláctica es la más grave, ocurre a solo unos pocos segundos después de la picadura, los síntomas son: dificultad para respirar, confusión, vómitos y disminución de la presión sanguínea, lo cual puede llevar a la pérdida de conciencia e incluso la muerte, debido al desencadenamiento de problemas circulatorios y respiratorios (Roodt et al., 2005; Lee, 2012; Ferreira et al., 2018). Por lo anterior, es importante que cuando se practica la apiterapia, previamente se realicen pruebas, por personal profesional calificado, para conocer el grado de sensibilidad que el paciente tenga hacia la apitoxina.

El uso de piquetes con abejas vivas sobre los pacientes se sustituye por cremas, linimentos, ungüentos o formas inyectables intradermales o subcutáneas, para imitar en estos últimos casos el acto del piquete de abeja; en estos productos se usa el veneno, el cual se obtiene de colectores que aplican estímulos eléctricos al insecto para facilitar la extracción de apitoxina, siendo cada vez más común este procedimiento (Mahmoud, 2012; Sanad y Mohanny, 2013). Desde hace varias décadas los extractores de apitoxina se mencionan en la literatura, en 1962 se diseñó un aparato para la extracción de apitoxina, el cual fue patentado en 1965 en los Estados Unidos por Palmer (1965), quien propuso un dispositivo que primeramente atrapa abejas adultas de una colmena, las cuales contienen una mayor cantidad de veneno, para posteriormente aplicarles descargas eléctricas que obligan a las abejas a aguijonear una capa plástica de silicón en cuya parte inferior es recolectado el veneno. En años posteriores, se han propuesto diferentes diseños, pero manteniendo el fundamento de estimular a las abejas a picar con base en impulsos eléctricos, y con mejoras adicionales como la utilización de células solares en sustitución de las baterías como fuente de poder (Nam-Cheol et al., 2011).

Después del diseño de los colectores de apitoxina, varios investigadores se dieron a la tarea de evaluar la calidad del veneno colectado, o si su cosecha influye negativamente en el vigor de la colonia de abejas. Se encontraron diferencias en la calidad del veneno según el sitio de recolección en la trampa (Abrantes et al., 2017), y se ha concluido que la cosecha de veneno en abejas carniolas e italianas no afecta el desarrollo de la cría dentro de la colonia (Omar, 2017).

La presente investigación fue planteada con el objetivo de estimar el potencial de producción anual de apitoxina, recolectada mediante un equipo automatizado que funciona con impulsos eléctricos, en una explotación apícola localizada en Irapuato, Guanajuato, México.

\section{Materiales y métodos}

La presente investigación se desarrolló en un apiario comercial que poseía treinta colmenas, ahí se tuvieron abejas de la raza italiana (nivel de pureza de 90 a $95 \%$ ) de la especie A. mellifera, ubicado en la comunidad de El Copal, del municipio de Irapuato, Guanajuato, México, localizado a $20^{\circ} 45^{\prime} 1,8^{\prime \prime}$ de latitud norte y $101^{\circ} 19^{\prime} 35,8^{\prime \prime}$ de longitud oeste, con una altitud de $1757 \mathrm{msnm}$, en donde predomina una vegetación de tipo pastizal mezclado con arbustos de diversas especies que alternan con plantas silvestres anuales, mezquites y cazahuates; a poca distancia del apiario se encuentran también campos agrícolas se siembran con cereales y hortalizas. Durante el período que abarcó este trabajo, los promedios de las condiciones climáticas fueron: $30,6{ }^{\circ} \mathrm{C}$ de temperatura máxima, $5,8^{\circ} \mathrm{C}$ de temperatura mínima, 53,3\% de humedad relativa y $1,9 \mathrm{~km} \cdot \mathrm{h}^{-1}$ de velocidad del viento, mientras que, precipitaron un total de 227,6 mm de lluvia (Fundación Guanajuato Produce A.C., 2018). Adicionalmente de la miel como producto principal, se cosechó también apitoxina, para ayudar a incrementar la rentabilidad de la explotación, estimándose mediante el presente estudio el potencial de producción de este veneno.

Se utilizó un colector de apitoxina marca Chivilcoy-Industria Argentina ${ }^{\circledR}$, el cual provee impulsos eléctricos de $10 \mathrm{~s}$ de duración a un voltaje de $12 \mathrm{v}$, seguidos de un tiempo sin estimulación eléctrica con $10 \mathrm{~s}$ de descanso; cada 
una de las cinco rejillas que integran el colector se colocaron en un igual número de colmenas, donde permanecieron funcionando durante $20 \mathrm{~min}$, después de lo cual el veneno ya cristalizado y capturado sobre el vidrio recolector, se raspó y pesó en una balanza analítica para su cuantificación. La cosecha de veneno se repitió cada quince días con las mismas colmenas, solo fue interrumpida en los meses cuando se tuvieron flujos de néctar abundantes (octubrenoviembre y marzo-abril), con la finalidad de no afectar la producción de miel. La primera colecta se realizó el 15 de agosto de 2016 y la última el 15 de junio de 2017, diez meses de cosecha en total.

Dos meses antes de iniciar la presente investigación, las colonias utilizadas tuvieron un cambio de reinas, las cuales fueron adquiridas en un criadero de reconocido prestigio, donde, entre otras cosas, cuidan no introducir genes de africanización para lograr reinas que den origen a abejas obreras con un comportamiento dócil.

Como consecuencia de una infestación natural del ácaro Varroa destructor en todas las colmenas del apiario, se dividieron las cinco colmenas bajo estudio en dos grupos diferenciados por el nivel de presencia de la plaga; dos colmenas con alta invasión del ácaro (con una media de 23,84 \% de aparición) y tres colmenas con baja invasión con un 9,33\% en promedio. Para evitar que esta plaga colapsara a las colmenas, el 12 de enero de 2017 se aplicó el piretroide flumetrina al $0,054 \%$, colocando cuatro tiras por colmena por cuarenta días, logrando un control efectivo al reducir la infestación a 2,08 \% en el grupo de mayor invasión y a 1,84 \% en el otro grupo. Aun con estos niveles de reducción de la plaga, las colonias con la infestación más intensa tardaron en recuperarse, permaneciendo débiles durante este estudio, lo que permitió su comparación con las colmenas con menor infestación, las cuales mostraron mayor energía y actividad, al ser poco afectadas por este ácaro.

Para comparar la producción de apitoxina en mg de cinco colmenas (cinco unidades experimentales) seleccionadas al azar durante trece fechas de medición, se utilizó la prueba no paramétrica de medidas repetidas. Para realizar la comparación del rendimiento de veneno en mg en seis meses de extracción, con base en tres colmenas con poca presencia de varroasis, se utilizó el análisis de varianza de una vía.

\section{Resultados}

Al momento de activar el colector de veneno, las abejas iniciaron un comportamiento de agresividad mientras que circulaban en su paso por la rejilla electrificada, como era de esperase; por esta razón muchas abejas comenzaron a salir de la colmena y se fueron aglomerando alrededor de la piquera, lo que permitió cosechar mayor cantidad de veneno; esto reflejó un comportamiento defensivo de las abejas, alterando momentáneamente la conducta de la colonia. Al quitar el colector de veneno después de 20 min de estar activado, las abejas regresaron su actividad normal.

La recolección de apitoxina realizada quincenalmente, permitió una alteración menor de la conducta normal de las abejas, comparado a que se hubiera hecho con mayor frecuencia; adicionalmente, al suspenderla durante los dos periodos en que sucedieron acopios abundantes de néctar, las abejas pecoreadoras entraron y salieron libremente por la piquera sin la interrupción que les provocaba el colector de veneno. Del 15 de octubre al 30 de noviembre de 2016 ocurrió el primer flujo de néctar, como consecuencia de la floración de todas las especies vegetales anuales que surgen, debido a la temporada de lluvias de la región, dando lugar a una cosecha de miel multifloral; tampoco se recolectó veneno del 15 de marzo al 30 de abril de 2017, ya que se presentó la floración de los árboles conocidos como mezquites (Prosopis juliflora), originando una cosecha de miel monofloral. Aunque el rendimiento de miel no formó parte de las variables medidas en la presente investigación, se observó que ambas cosechas fueron similares al rendimiento obtenido en las colmenas del mismo apiario que no formaron parte de este experimento.

Bajo las condiciones en las que se manejó el colector de veneno en la presente investigación, se obtuvo un total de 681,33 mg de apitoxina por colmena durante seis de los diez meses que duró la investigación (Cuadro 1), ya 
Cuadro 1. Producción de apitoxina en un apiario ubicado en El Copal, municipio de Irapuato, Guanajuato, México, durante diez meses de recolección (15 de agosto de 2016 a 15 de junio de 2017), contrastando colmenas con alta (1 y 2) y baja (3, 4 y 5) infestación de V. destructor.

Table 1. Production of apitoxin in an apiary located in El Copal, municipality of Irapuato, Guanajuato, Mexico, during ten months of collection (August 15, 2016 to June 15, 2017), contrasting hives with high (1 and 2) and low (3,4 and 5) infestation of V. destructor.

\begin{tabular}{|c|c|c|c|c|c|c|}
\hline \multirow{3}{*}{$\begin{array}{c}\text { Fecha de } \\
\text { colecta }\end{array}$} & \multicolumn{5}{|c|}{ Producción de apitoxina (mg.colmena-1) } & \multirow{3}{*}{$\begin{array}{c}\text { Promedio } \pm \\
\text { desviación } \\
\text { estándar }\end{array}$} \\
\hline & \multicolumn{2}{|c|}{ Alta infestación de $V$. destructor } & \multicolumn{3}{|c|}{ Baja infestación de $V$. destructor } & \\
\hline & Colmena 1 & Colmena 2 & Colmena 3 & Colmena 4 & Colmena 5 & \\
\hline $15 / 08 / 2016$ & 63,70 & 51,61 & 68,11 & 53,38 & 61,56 & $59,67 \pm 6,99$ \\
\hline $31 / 08 / 2016$ & 61,61 & 58,12 & 60,92 & 67,16 & 66,89 & $62,94 \pm 3,95$ \\
\hline $15 / 09 / 2016$ & 55,20 & 53,17 & 63,78 & 52,74 & 58,31 & $56,64 \pm 4,56$ \\
\hline $30 / 09 / 2016$ & 51,00 & 56,49 & 58,51 & 63,44 & 62,76 & $58,44 \pm 5,07$ \\
\hline $15 / 10 / 2016$ & \multicolumn{5}{|c|}{ Flujo de néctar (miel multifloral) } & \\
\hline $31 / 10 / 2016$ & \multicolumn{5}{|c|}{ Flujo de néctar (miel multifloral) } & \\
\hline $15 / 11 / 2016$ & \multicolumn{5}{|c|}{ Flujo de néctar (miel multifloral) } & \\
\hline $30 / 11 / 2016$ & \multicolumn{5}{|c|}{ Flujo de néctar (miel multifloral) } & \\
\hline $15 / 12 / 2016$ & 23,45 & 27,11 & 55,30 & 58,20 & 63,45 & $45,50 \pm 18,73$ \\
\hline $31 / 12 / 2016$ & 27,91 & 28,02 & 66,49 & 63,69 & 59,11 & $49,04 \pm 19,42$ \\
\hline $15 / 01 / 2017$ & 19,23 & 24,70 & 59,95 & 60,63 & 67,24 & $46,35 \pm 22,52$ \\
\hline $31 / 01 / 2017$ & 24,12 & 23,07 & 63,77 & 67,50 & 67,68 & $49,23 \pm 23,45$ \\
\hline $15 / 02 / 2017$ & 20,46 & 28,98 & 62,67 & 59,98 & 62,30 & $46,88 \pm 20,48$ \\
\hline $28 / 02 / 2017$ & 26,18 & 26,61 & 62,13 & 61,31 & 65,36 & $48,32 \pm 20,07$ \\
\hline $15 / 03 / 2017$ & \multicolumn{5}{|c|}{ Flujo de néctar (miel monofloral de mezquite) } & \\
\hline $31 / 03 / 2017$ & \multicolumn{5}{|c|}{ Flujo de néctar (miel monofloral de mezquite) } & \\
\hline $15 / 04 / 2017$ & \multicolumn{5}{|c|}{ Flujo de néctar (miel monofloral de mezquite) } & \\
\hline $30 / 04 / 2017$ & \multicolumn{5}{|c|}{ Flujo de néctar (miel monofloral de mezquite) } & \\
\hline $15 / 05 / 2017$ & 32,83 & 34,33 & 67,31 & 55,78 & 59,29 & $49,91 \pm 15,49$ \\
\hline $31 / 05 / 2017$ & 35,61 & 37,42 & 68,18 & 62,80 & 65,99 & $54,00 \pm 16,09$ \\
\hline $15 / 06 / 2017$ & 38,82 & 40,01 & 64,22 & 65,18 & 63,83 & $54,41 \pm 13,71$ \\
\hline Promedio: & $36,93 \pm 15,83$ & $37,66 \pm 12,93$ & $63,18 \pm 3,87$ & $60,91 \pm 4,80$ & $63,37 \pm 3,19$ & \\
\hline
\end{tabular}

que en los cuatro meses restantes ocurrieron los acopios de néctar mencionados y que dieron por consecuencia las dos cosechas de miel. En total, se recolectó veneno trece veces, iniciando el 15 de agosto de 2016 y terminando el

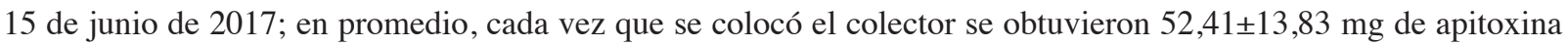
por colmena, cantidad de veneno que logró acumularse durante los 20 min que el colector estuvo activado en cada

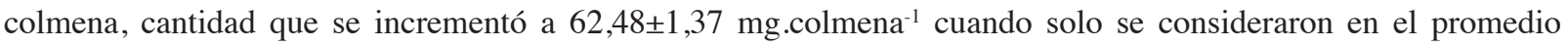
aquellas colonias libres del ácaro $V$. destructor. La menor producción de apitoxina correspondió a una de las colmenas debilitadas por la infestación de V. destructor, con 19,23 mg (para el 15 de enero en la colmena 1), mientras que, la mayor producción fue de $68,18 \mathrm{mg}$ (para el 31 de mayo en la colmena 3) en una de las colmenas con menor infestación de dicho ácaro. 
La alta infestación de $V$. destructor en las colmenas 1 y 2 utilizadas en la presente investigación, causó una reducción en el número de abejas obreras en la colonia, lo que fue un factor determinante en la reducción del rendimiento de apitoxina cosechada, lo cual se corroboró con la prueba de medidas repetidas aplicada para comparar las cinco colmenas, y obtener diferencias estadísticas significativas ( $\left.\mathrm{F}=26,68, \mathrm{P}=0,0001^{* *}\right)$. Posteriormente se utilizó la prueba de t para el contraste de medias (Figura 1), resultando dos grupos estadísticos, en el grupo "B" se ubicaron las colmenas debilitadas 1 y 2 , con medias de veneno de 36,93 y $37,66 \mathrm{mg}$, respectivamente; mientras que en el grupo "A", se encontraron las colmenas vigorosas 3 con 63,18 mg, 4 con 60,91 mg y 5 con 63,37 mg de apitoxina en promedio. Es de suponer que cualquier otro factor que debilite las colonias de abejas, producirá un efecto similar en la producción de apitoxina.

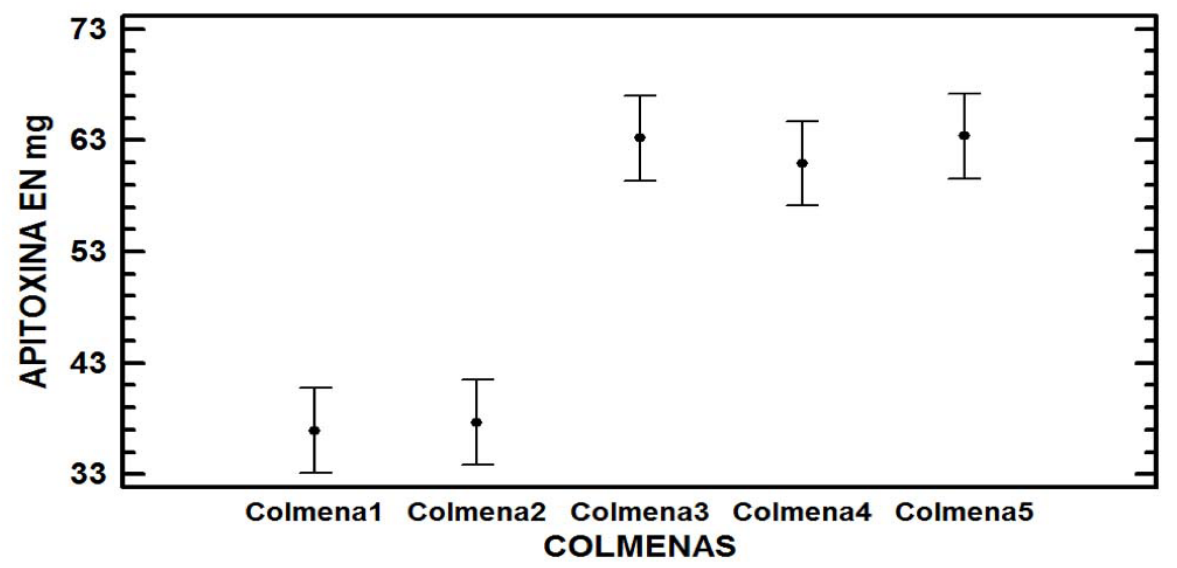

Figura 1. Prueba de t para comparar la producción de apitoxina de cinco colmenas en en el periodo de agosto de 2016 a junio de 2017 , en Irapuato, Guanajuato, México.

Figure 1. Test of t to compare the production of apitoxin from five hives in the period from August 2016 to June 2017, in Irapuato, Guanajuato, Mexico.

\section{Discusión}

Una preocupación del apicultor cuando decide recolectar apitoxina, es que no se vea afectada la producción de miel, razón por la cual se evitó colocar el colector de veneno durante los dos principales flujos de néctar que ocurrieron durante el periodo en que se desarrolló esta investigación, ya que la cosecha de apitoxina puede desencadenar una reacción de defensa en la colonia de abejas causándoles estrés, y provocando cambios en su comportamiento que podrían influir en las actividades rutinarias de la colonia, entre las que se puede incluir el acopio de néctar (Onari et al., 2016). Por esta razón, y de acuerdo con los resultados obtenidos, en los períodos de recolección abundante de néctar, se sugiere dejar de colectar apitoxina para cosechar lo que normalmente se produce de miel, la cual sigue siendo el producto de interés principal entre los apicultores; este criterio también fue considerado por Bahreini et al. (2000), quienes obtuvieron $838 \mathrm{mg}$ de veneno por colmena, obtenido quincenalmente durante seis meses, sin observar ningún efecto adverso en la cosecha de miel; por otra parte, Zhou et al. (2003) cuantificaron una reducción de hasta $49,9 \%$ en la producción de miel cuando las colmenas se sometieron a una cosecha de veneno más intensiva, es decir, cada tercer día.

La máxima producción de apitoxina obtenida fue de 68,18 mg.colmena ${ }^{-1}$, sin embargo, en otras investigaciones se han conseguido rendimientos tanto más altos como más bajos; Sanad y Mohanny (2013) obtuvieron de 31 a $185 \mathrm{mg}$ de veneno por colmena por día, aunque con tiempos de recolección mayores a los utilizados en la presente 
investigación, mientras que Modanesi et al. (2015) lograron su máxima producción (49,1 mg) por la mañana (9 am) durante $60 \mathrm{~min}$ de recolección. No hay coincidencia en la literatura sobre las condiciones óptimas para la producción de apitoxina, sugiriéndose que las condiciones específicas en cada lugar condicionan los posibles rendimientos de veneno a obtener.

Algunas colonias de abejas manejadas en este estudio se vieron debilitadas por una alta infestación del ácaro $V$. destructor, y por consecuencia, producir cantidades de veneno menores en comparación con colonias vigorosas donde dicho ácaro se presentó en bajos niveles de infestación. Esto permitió deducir que el vigor de la colonia influyó significativamente en la producción de apitoxina. A partir de enero de 2017, las colonias de las colmenas 1 y 2 se debilitaron, lo que provocó la disminución en la producción de veneno de ambas colmenas a partir de esa fecha. Desde hace varios años se han mencionado los efectos de $V$. destructor como plaga de las abejas, entre los que se pueden mencionar muerte de larvas y ninfas, deformaciones de alas, patas, abdomen y tórax en adultos, disminución de la potencia sexual de los zánganos, reducción en el tiempo de vida y el tamaño de las obreras, mayor vulnerabilidad a enfermedades, y como consecuencia, las abejas al sentirse débiles, trabajan menos y mal, o son expulsadas de la colmena, lo que provoca un impacto negativo en la colonia (Botta et al., 2004; Zemene et al., 2015; Piou et al., 2016).

Las colonias que presentaron baja incidencia de V. destructor (colmenas 3, 4 y 5), no mostraron diferencias estadísticas en la producción de apitoxina ( $\mathrm{F}=0,89, \mathrm{P}=0,4988 \mathrm{NS}$ ), con promedios entre 59,92 a 64,46 mg, lo que contrasta con otros estudios que encontraron que algunos meses del año son mejores que otros para la cosecha de veneno de abejas (Sanad y Mohanny, 2013). Por lo tanto, se puede deducir que en la región donde se desarrolló este estudio, cualquier época del año es propicia para que se recolecte veneno, pero su mayor producción está condicionada al grado de salud y vigor de la colonia.

Las tres colmenas vigorosas $(3,4$ y 5 ) fueron estadísticamente iguales en cuanto a la cantidad de veneno producido, debido a que, en este caso, la población de abejas que entraron y salieron por la piquera es alta, por lo que se obtuvo un mayor flujo de abejas obreras que pasaron a través del colector de apitoxina, lo que aumentó la producción de veneno en comparación con colmenas con colonias débiles; se puede argumentar que el mantener colonias saludables y ampliar la recolección de veneno a los doce meses del año, pero interrumpiendo dicha cosecha cuando se presenten flujos abundantes de néctar, para no demeritar la producción de miel, el potencial de producción de apitoxina se incrementaría a 999,74 mg.colmena ${ }^{-1}$; esta cantidad podría parecer poca, pero se debe considerar que se necesitan al menos un millón de piquetes de abeja para recuperar un gramo de veneno seco (Mahmoud, 2012), y según Dotimas y Hider (1987), un gramo de veneno puede ser recolectado de veinte colmenas en un lapso de dos horas.

Se sugiere que la cosecha de apitoxina no sea más frecuente a como se efectuó en este estudio (quincenalmente y por solo 20 min por vez), lo que en parte concuerda con otras investigaciones, donde los mejores resultados se han encontrado al cosechar el veneno cada catorce días (Rybak et al., 1995; Rybak y Skubida, 2007); el hacerlo con mayor frecuencia podría interferir con las condiciones de la colonia y alterar el desarrollo de la cría (Onari et al., 2016; Omar, 2017), o con un incremento en la tendencia de las abejas a abandonar la colmena, como ocurrió en el trabajo de Onari et al. (2016), cuando las colonias fueron sometidas a la cosecha de apitoxina en forma quincenal por una hora; aunque contrasta con el trabajo de Sanad y Mohanny (2013), quienes no encontraron efectos colaterales en la actividad de las obreras y la producción de miel cuando se cosechó el veneno cada tercer día.

\section{Conclusiones}

Se cuantificó la producción potencial de apitoxina para la región apícola donde se desarrolló esta investigación, con el empleo de colectores basados en estímulos eléctricos, los cuales tienen la ventaja de mantener con vida a las 
abejas, proponiéndose como un producto apícola adicional que permitiría incrementar la rentabilidad del apiario, cuyo principal producto a cosechar es la miel; al no morir las abejas e interrumpiendo la recolección de apitoxina durante los períodos de acopio importante de néctar, se mantiene sin alteración el número y la actividad normal de las abejas pecoreadoras, reduciendo la posibilidad de obtener menor cosecha de miel.

\section{Literatura citada}

Abrantes, A.F., R.T. Cavalcante, L.A.B. Sales, and C.M. Tejo. 2017. Honey bee venom: influence of collection on quality and cytotoxicity. Cienc. Rural 47(10):1-4. doi:10.1590/0103-8478cr20160486

Bahreini, R., K. Fakhimzadeh, J. Nowzary, and G.A. Jehzati. 2000. Design and construction of a venom collecting electric cage and its effects on honey production in honey bee colonies. Iran. J. Agr. Sci. 31:333-339.

Botta, E., H. Carmenate, y P.E. De-la-Torre. 2004. Varroasis, peligrosa enfermedad de la abeja melífera. Fitosanidad 8(1):73-79.

Dotimas, E.M., and R.C. Hider. 1987. Honeybee venom. Bee World 68(2):51-70. doi:10.1080/0005772X.1987.11098915

Eze, O.B.L., O.F.C. Nwodo, and V.N. Ogugua. 2016. Therapeutic effect of honey bee venom. Int. J. Pharm. Chem. Biol. Sci. $4(1): 48-53$

Ferreira, M.T.L., D. Campelo-Batalha-Cox-Moore, D. Mancino-Da-Luz-Caixeta, M. Dos-Santos-Salú, C.V. Barreto-Farias, and Z.M.Almeida-de-Azevedo. 2018. Multiple bee stings, multiple organs involved: a case report. Rev. Soc. Bras. Med. Trop. 51:560-562. doi:10.1590/0037-8682-0341-2017

Fratellone, P.M., and G. Fratellone. 2016. Apitherapy products for medicinal use. J. Altern. Complement. Med. 22:1020-1022. doi: $10.1089 / \mathrm{acm} .2015 .0346$

Fundación Guanajuato Produce, A.C. 2018. Red de estaciones climáticas. Estación El Copal, municipio de Irapuato. Fundación Guanajuato Produce, A.C., MEX. http://www.estaciones.fundacionguanajuato.mx/export (consultado 12 ene. 2018).

Goodman, L.J. 2003. Form and function in the honey bee. International Bee Research Association, Cardiff, GBR.

Lee, K. 2012. Correlation between the constitution of sasang and sexual difference in the hypersensitive reaction of sweet bee venom. J. Pharmacopunct. 15(3):45-47. doi:10.3831/KPI.2012.15.005

Mahmoud, A. 2012. Studies on bee venom and its medical uses. Inter. J. Adv. Res. Technol. 1(2):1-15.

Modanesi, M.S., S.M. Kadri, P.E.M. Ribolla, D.P. Alonso, and R.O. Orsi. 2015. Period and time of harvest affects the apitoxin production in Apis mellifera Lineu (Hymenoptera: Apidae) bees and expression of defensin stress related gene. Sociobiology 62:52-55. doi:10.13102/sociobiology.v62i1.52-55

Nam-Cheol, C., L. Chae-Moon, and K. Choul-Goo. 2011. An experimental study on the bee venom collector using the photovoltaic system. J. Korean Sol. Energy Soc. 31(4):122-127. doi:10.7836/kses.2011.31.4.122

Omar, R.E.M. 2017. Effect of bee venom collection on the measurement of brood rearing activity of honey bee colony Apis mellifera L. Middle East J. Agric. Res. 6:409-414.

Onari, P., R. Zaluski, T.S. Bovi, and R.O. Orsi. 2016. Apitoxin harvest affects population development but not the hygienic behavior of African-derived honey bees. Sociobiology 63:688-692. doi:10.1007/s13592-017-0520-8

Palmer, D.J. 1965. Apparatus for the extraction of venom from bees. Patent No. 3.153.371. United States Patent Office, USA.

Piou, V., J. Tabart, V. Urrutia, J.L. Hemptinne, and A. Vétillard. 2016. Impact of the phoretic phase on reproduction and damage caused by Varroa destructor (Anderson and Trueman) to its host, the European honey bee (Apis mellifera L.). PLoS One. 11(4):e0153482. doi:10.1371/journal.pone.0153482 
Roodt, A.R., O.D. Salomón, T.A. Orduna, O.L.E. Robles, S.J.F. Paniagua, y C.A. Alagón. 2005. Envenenamiento por picaduras de abeja. Gac. Méd. Méx. 141:215-222.

Rybak, M., J. Muszynska, P. Skubida, and J. Marcinkowski. 1995. A technology for bee venom collection. Pszczelinicze Zeszyty Naukowe 39(S):223-231.

Rybak, M., and P. Skubida. 2007. Application of coupled electrical and sound stimulation for honey bee venom collection. J. Api. Sci. 51(2):63-66.

Sanad, R.E., and K.M. Mohanny. 2013. The efficacy of a new modified apparatus for collecting bee venom in relation to some biological aspects of honey bee colonies. J. Am. Sci. 9(10):177-182. doi:10.7537/marsjas091013.23

Schumacher, M.J., J.O. Schmidt, and W.B. Egen. 1989. Lethality of killer bee stings. Nature 337:413. doi:0.1038/337413a0

Shimpi, R., P. Chaudhari, R. Deshmukh, S. Devare, Y. Bagad, and M. Bhurat. 2016. A review: pharmacotherapeutics of bee venom. World J. Pharm. Pharm. Sci. 5:656-667. doi:10.20959/wjpps20167-7187

Sobral, F., A. Sampaio, S. Falcao, M.J. Queiroz, R.C. Calhelha, M. Vilas-Boas, and I.C. Ferreira. 2016. Chemical characterization, antioxidant, anti-inflammatory and cytotoxic properties of bee venom collected in Northeast Portugal. Food Chem. Toxicol. 94:172-177. doi:10.1016/j.fct.2016.06.008

Winston, M.L. 1987. The biology of the honey bee. Harvard University Press, Cambridge, MA, USA.

Zemene, M., B. Bogale, S. Derso, S. Belete, S. Melaku, and H. Hailu. 2015. A review on Varroa mites of honey bees. Acad. J. Entomol. 8(3):150-159. doi:10.5829/idosi.aje.2015.8.3.95259

Zhou, B., S. Zhang, C. Su, G. Zhou, B.F. Zhou, S.J. Zhang, C. Su, and G.H. Zhou. 2003. Effect of collection of venom by electric shocking on honeybee population, production of royal jelly and honey. Acta Agr. Univ. Jiangxi. 25(1):141-145. 